\title{
Gemälde fallen aus dem Rahmen. Akte der Bildaneignung in Manuel Mujica Láinez' Roman Un novelista en el Museo del Prado
}

\author{
Bettina Korintenberg
}

Faktualität und Fiktionalität sind zentrale Klassifikationskategorien, über die wir Bildern Bedeutung zumessen, sie funktionalisieren und mit ihnen agieren. Durch diese Differenz geben wir Bildern einen Rahmen und lenken dadurch unsere Wahrnehmung. Und doch sind Bilder weitaus komplexer, als dass sie sich einem solchen Oppositionsschema beugen würden. Dies führt der Roman Un novelista en el Museo del Prado des argentinischen Schriftstellers Manuel Mujica Láinez eindrucksvoll vor. Die fiktionale Narrativierung der faktualen Bildwerke im Prado als realreferentieller Ort $^{1}$ durch den Betrachter steht im Mittelpunkt der Handlung. Die Hauptfigur, der ,novelista', wird als Zeuge der nächtlichen Aktivitäten der Bildartefakte des Prados, wenn diese zum Leben erwachen und zu interagieren beginnen, eingeführt und ist so der Wahrhaftigkeit verpflichtet. Über die Bildszenarien aus historischen, mythologischen und religiösen Figuren der europäischen Kultur sind mehrere Reflexionsschritte geleitet: Durch den Aufbruch der gerahmten Bilder und damit der bildimmanenten Narration kommt es zu einer Infragestellung historisch fixierter Wirklichkeitskonstruktionen, die durch diese repräsentiert werden. Verstärkt wird dieser Eindruck durch die im Roman geleistete implizite Reflexion über den Status und die Funktionalisierung von ikonischen Artefakten und deren Präsentation innerhalb der paradigmatischen Institution Museum und der dort suggerierten Narration, die strukturierend und bekräftigend auf die Wahrnehmung der äußeren Realität zurückwirkt.

Im Roman kommt es zu komplexen Verschränkungen von Faktualität und Fiktionalität, die maßgeblich über das Bild geleitet werden. In diesem Artikel sollen diese Prozesse theoretisch und textanalytisch nachvollzogen werden. Die Imagination übernimmt bei Mujica Láinez die zentrale Funktion, die Offensichtlichkeit kategorialer Trennungen von faktual und fiktional zu unterlaufen. Bei der Betrachtung der materiellen Bildwerke - der Gemälde und Skulpturen - verschachteln sich die Bilder, wie sie im tatsächlichen Raum des Museums sichtbar sind mit den mentalen, eigentlich unsichtbaren Bildern des Rezeptionssubjekts. Der Roman nimmt sich auf diese Weise der unverkennbaren Heterogenität des Bildbegriffs an. Über

1 Nach Linda Hutcheon ließe sich Mujica Láinez’ Roman als „historiographic metafiction“ lesen, die sich durch das Zusammenkommen historischer Ereignisse und Personen bei gleichzeitiger Reflexion über diese charakterisiert. („By this I mean those well-known and popular novels which are both intensely self-reflexive and yet paradoxically also lay claim to historical events and personage." Hutcheon 1988: 5) 
die Gegensätze Sichtbarkeit - Unsichtbarkeit, Repräsentation - Präsenz weist der Roman auf die Fragilität einer Klassifikation von Bildern und der durch sie repräsentierten Wirklichkeitsvorstellungen hin. In den folgenden Ausführungen spielen deswegen bildtheoretische Überlegungen eine zentrale Rolle.

Was den Leser im Roman erwartet, sind alles andere als vertraute Bilder, die man mit dem fest im kulturellen Gedächtnis verankerten Ort des Prados, dessen Ausstellungsstücke von der kulturellen Blüte und Hegemonie Europas zeugen und erzählen, in Verbindung bringen könnte. Kontextuell zu beachten ist hierbei die postkoloniale Konstellation Lateinamerika - Europa, innerhalb derer sich der Roman verortet und die sich nochmals im Plot, ein Fremder aus der Ferne erkundet den Prado als eines der bedeutendsten europäischen Museen, wiederspiegelt. Zwar steht diese Thematik nicht im Mittelpunkt dieses Artikels, am Rande jedoch wird sie immer wieder auftauchen, denn für das Gesamtverständnis des Romans ist sie unabdingbar.

\section{Bilder. Im Anderswo zwischen Faktualität und Fiktionalität}

Das Bild entzieht sich einer klaren Definition. Der Roman nimmt sich der komplexen und heterogenen Kategorie Bild an, indem sehr unterschiedliche Bildbegriffe formuliert werden: das Gemälde an der Wand, die Evokation eines Bildes in Gedanken, ein Textbild. „Was ist ein Bild?“ (Boehm 2001) - diese Frage stellt der paradigmatische Sammelband, herausgegeben von Gottfried Boehm, und zielt damit auf den Kern der Bild-Problematik. Bis heute bleibt sie unbeantwortet. Dass die Antwort nicht in der einfachen Unterscheidung von Sichtbarkeit und Unsichtbarkeit zu finden ist, liegt nahe und spiegelt sich in den Ansätzen der Bildwissenschaften. ${ }^{2}$ Schon früh beschreitet Boehm einen Weg, um der Eigenlogik und Wirkkraft der Bilder auf die Spur zu kommen, der ihn hinter eine logozentrische, hin zu einer autonomen, bildlich organisierten Wahrnehmung führt. ${ }^{3}$ Hans Belting nimmt den Gedanken einer eigenlogischen Wirkmacht von Bildern auf und prägt einen anthropologisch-pragmatischen Bildbegriff, wie er in unserem Zusammenhang wichtig wird. ${ }^{4}$

2 Im Zentrum der Bildwissenschaften stehen Fragen nach Bildpraxen, also einem Handeln mit und durch Bilder, sowie deren Wirkung und Wirkmacht, die nicht nur durch deren soziokulturelle Einbettung zu erklären ist, sondern als eigenständige, vom Bildlichen ausgehende Energie gedacht wird. Diese Aspekte überschreiten die Sichtbarkeit der visuellen Oberfläche des Bildes und zielen auf dessen anthropologische und kulturelle Konstituenten. Einen guten Überblick über die Entwicklung bildtheoretischer Fragestellungen gibt der von Klaus Sach-Hombach herausgegebene Sammelband Bildtheorien.

3 Wegweisend ist sein Aufsatz „Zu einer Hermeneutik des Bildes“ von 1978, in dem er das Konzept der ikonischen Differenz entwickelt, um die bildeigene, explikative Wirkkraft des Bildes, dessen Bildenergie, zu ergründen.

4 In der Bild-Anthropologie entwickelt Belting schlaglichtartig ,Entwürfe‘ für eine interdisziplinäre und interkulturelle Bildwissenschaft, bei denen der Körper als Grundprinzip eine zentrale Rolle spielt. Der Körper fungiert maßgeblich als Umschlagplatz und Resonanz- 
Für die angestellten Überlegungen ist zweierlei zentral: 1. Bilder existieren nicht einfach isoliert im Raum. Sie sind an ihre Rezeption gebunden und stehen stets innerhalb eines spezifischen Kontextes. ${ }^{5}$ Sie werden vom kollektiven Imaginären ${ }^{6}$ sowie der Imagination des Subjekts strukturiert. Bilder sind demnach mehr als ihre Sichtbarkeit, sie umgibt ein imaginärer Umraum ${ }^{7}$, der ihre Bedeutung grundiert. „Wir müssen die Augen schließen, um zu sehen, während der Akt des Sehens uns auf eine Leere verweist und auf sie hin öffnet, welche uns anblickt, uns betrifft und in einem bestimmten Sinne konstituiert" (Didi-Huberman 1999: 13). Die Leere der Oberfläche verweist bei Didi-Huberman auf eine Fülle im Unsichtbaren. Und so lebt die Sichtbarkeit aus dem nicht sichtbaren Abwesenden. An- und Umlagerungen, die im Bild mit eingeschlossen sind, werden im Rezeptionsakt aktiv und weisen auf die das Subjekt konstituierenden Muster hin. ${ }^{8}$ 2. Bilder unterliegen pragmatischen Rahmungen, die uns in unserer Wahrnehmung dieser Bilder beschränken und lenken. Mit Bedeutung aufgeladen, sind sie Teil symbolischer Handlungen und nicht nur Teil individueller Subjektivierungsweisen, sondern wirken intersubjektiv. Wir handeln durch und mit Bildern, aber Bilder handeln auch mit uns. ${ }^{9}$ Bilder und Bildwelten sind reziprok angelegt: Wir funktionalisieren, institutionalisieren und ritualisieren Bilder, gleichzeitig wirken sie auf uns ein, was immer ein nur teilweise zu antizipierender und zu kontrollierender Prozess ist. In der Imagination des Einzelnen kann die pragmatische Rahmung gesprengt werden, indem sie neu perspektiviert wird.

raum mentaler und physischer Bilder. Belting bezieht sich hierbei sowohl auf den Körper des Betrachters und dessen Umgang mit den Bildern, als auch auf den Körper des Bildes, der sich in dessen Materialität manifestiert und körperhafte Wirkungen auf den Betrachter entfaltet. Damit schließt Belting an seine prominenten Überlegungen zur Bildgeschichte, die er 1990 in Bild und Kult formulierte, an und führt sie weiter.

5 Zentral ist hier die These von der Historizität der Wahrnehmung, wie sie Walter Benjamin in Das Kunstwerk im Zeitalter seiner technischen Reproduzierbarkeit formuliert. Nach Benjamin ist Wahrnehmung historisch bedingt und unterliegt demnach gesellschaftlichen Entwicklungen durch die Zeit, wie etwa die Wahrnehmung von Kunstwerken entscheidend mittels neuer Medien geprägt wird.

6 In Cornelius Castoriadis' Theorie Gesellschaft als imaginäre Institution hat das Imaginäre eine kollektive Dimension: Es bedeutet die innerhalb einer Gesellschaft möglichen Vorstellungen (Einbildungskraft), die den gesellschaftlichen Institutionen zugrundeliegenden Ideen über die Welt. Subjektive Ansichten entstehen demnach nicht im luftleeren Raum, sondern in Anlehnung an die allgemein herrschenden Vorstellungsmuster der jeweiligen Gesellschaft.

7 Didi-Huberman spricht in diesem Zusammenhang von Leere, Leerraum oder auch unausweichlichem Volumen im Sinne eines Rauminhalts (vgl. Didi-Huberman 1999: 11-14).

8 In ähnlicher Weise wie Didi-Huberman formuliert dies auch Ludger Schwarte, wenn er von der doppelten Disposition des Bildes spricht: als „ein Fenster auf ein ,Außen““ sowie als „eine Spiegelung, ein Zerrspiegel der Transfigurationen, welche sich dadurch, dass es [das Bild] sich zu sehen gibt, im Betrachter vollzieht" (Schwarte 2005: 297).

9 Die Reziprozität des Bildlichen wurzelt nach Christoph Wulf und Jörg Zirfas in der Performativität als zentraler Kategorie des menschlichen Handelns: „Menschliche Performanz erzeugt Bilder und wird durch Bilder hervorgebracht. [...] Bilder sind also nicht nur Ergebnisse der Performativität kulturellen Handelns und Verhaltens, sondern sie erzeugen sie auch" (Wulf/Zirfas 2005: 7). 
Bilder sind immer auch Bildhandlungen, die ein komplexes Ineinander innerer und äußerer Bilder ergeben. „Äußere Bilder gehen auf innere, und innere Bilder auf äußere zurück“ - sie seien unauflösbar und wechselseitig miteinander verschränkt so Wulf (Wulf 2005: 39). ${ }^{10}$ Diese Verschränkung von innen und außen, von kollektivem Imaginären und subjektiver Imagination legt Mujica Láinez dar. Sein Roman lässt sich als textuelle performative Bildbeschreibung lesen, bei der die Imagination als zentrale Schaltstelle zwischen Faktualität und Fiktionalität fungiert. Die Romanhandlung veranschaulicht, wie Bildartefakte, Werke mit realweltlichen Referenten, von der Imagination im Akt der Rezeption bearbeitet, narrativ ein- und um(ge)woben werden. Die Trennschärfe von Faktualität und Fiktionalität scheint dabei in der Interferenz von kollektivem Imaginären und individueller Imagination als Emergenzphänomen zu verschwimmen.

In der narrativen Ausformung gewinnen die ikonischen Artefakte eine andere, neue Art interpretativer Realisierung. Bei der Rezeption des Textes kann diese realisierte Interpretation wiederum modellierend auf das kollektive Imaginäre des Lesers rückwirken. Im Folgenden sollen die Umwandlungsprozesse der narrativen, fiktionalen Ausgestaltung des Erlebens faktualer, historischer Bilder in Un novelista en el museo del Prado schrittweise nachvollzogen werden: Unter dem Punkt „Bilder rahmen. Bildbannung und enthemmte Bildenergie“ geht es zunächst um die konkrete Einbettung und Fixierung der ikonischen Artefakte innerhalb der Institution Museum und deren spannungsvolle Loslösung in der textuellen Darstellung der Bildrezeption durch den Protagonisten. Unter „Bilder treffen. Blicken und angeblickt werden" stehen bildtheoretische Überlegungen zur kommunikativen Dimension von Bildern im Mittelpunkt, die Mujica Láinez' Roman maßgeblich prägen. Der Blick des betrachtenden ,novelista' aktiviert die Bilder und entfacht deren Gegenblick, was sich im Roman in der narrativen Neukonfiguration der Bildwerke abzeichnet. Wie diese Narrativierung genau aussieht, welche textuellen Modi und Strategien der Text anwendet, wird im vorletzten Punkt „Die Präsenzerfahrung der Bilder. Ein Spektakel der Körper und Töne“ analysiert. Abschießend werden unter „Zusammenschau und Ausblick“ die gewonnenen Erkenntnisse hinsichtlich Struktur und rezeptionsästhetischer Wirkung des Romans, der sich als textuelle performative Bildbetrachtung lesen lässt, in Bezug auf dessen gesellschaftskulturelle Implikationen diskutiert und auf die Problematik von Faktualität und Fiktionalität hin reflektiert.

10 Die unauflösbare Verschränkung innerer und äußerer Bilder als deren Wesen liegt nach Belting in ihrer „anthropologische[n] Fundierung“. „Wir leben mit Bildern und verstehen die Welt in Bildern. Dieser lebende Bildbezug setzt sich gleichsam in der physischen Bildproduktion fort, die wir im sozialen Raum veranstalten. Sie verhält sich zu den mentalen Bildern wie Frage und Antwort, um eine vorläufige Formulierung anzuwenden“" (Belting 2001: 11-12). 


\section{Bilder rabmen. Bildbannung und enthemmte Bildenergie}

Der Prado, in dem die Romanhandlung spielt, konstituiert die gesellschaftlich kulturelle Rahmung und perspektiviert die gerahmten oder auf Podesten platzierten Bildwerke, die dadurch auf zweifache Weise in ihrer Wahrnehmung fixiert sind. Denn im Museum werden Werke, und damit Wissen, gestaltet und in Bezug auf ein kollektives Imaginäres hin geordnet. Dies entspricht dem Prinzip der Repräsentation. Die Repräsentationsmacht speist sich insbesondere daraus, dass die Gegenstände nicht nur in einem Ähnlichkeitsverhältnis stehen, sondern an der Macht des Repräsentierten partizipieren und dessen gesellschaftlich fixierte Geschichte kondensiert in sich tragen. ${ }^{11}$ Die im Prado ausgestellten kanonisierten Werke zeugen von der Überlegenheit der europäischen Kunst- und Kulturgeschichte. Bei den Werken, die in den Text aufgenommen sind, handelt es sich hauptsächlich um Gemälde und einige wenige Skulpturen der bekanntesten europäischen Künstler wie Dürer, Bosch, Velázquez, Goya und Fra Angelico. Sie stehen innerhalb eines europäischen Kulturkanons paradigmatisch für religiöse, gesellschaftspolitische und ästhetische Diskurse. Vor den Augen des ,novelista', des Romanciers, der dem Leser als derjenige vorgestellt wird, der die folgenden Geschehnisse erlebt und erzählt, ${ }^{12}$ verlassen die Figuren der Bilder und Skulpturen des Nachts ihren Rahmen; sie beginnen, zu agieren und interagieren. ${ }^{13}$ Die Bildbetrachtung, die der Text vollzieht, entwirft eine Art Bildercollage. In einer Reihe episodischer Bühnenstücke kommen kurze Geschichten zur Aufführung, in denen sich die Protagonisten der kanonisierten Werke einer europäischen Kunst- und Kulturgeschichte von einer ganz anderen Seite zeigen, als die, die wir eigentlich von ihnen kennen.

So wie Mujica Láinez das Museum narrativ ausgestaltet, schließt er an das Konzept der Heterotopie nach Foucault an und geht zugleich darüber hinaus. Foucault bestimmt Heterotopien als „Gegenplatzierungen oder Widerlager, tatsächlich realisierte Utopien, in denen die wirklichen Plätze innerhalb der Kultur gleichzeitig repräsentiert, bestritten und gewendet sind“ (Foucault 1991: 39). Wie

11 Krzysztof Pomian bezieht sich hier zwar auf vormoderne Sammlungen, die allgemeinen Charakteristika haben aber durchaus übergreifende Gültigkeit: Sammlungsgegenstände können verschiedene Personen- bzw. Personengruppen repräsentieren. Sie stehen etwa für das Ansehen der Götter, denen ein Tempel geweiht ist und verweisen auch auf diese als ,Personen' oder sie repräsentieren Teilnehmer von Ereignissen, Personen mit bestimmten Fähigkeiten, etc. Bedeutsam ist dabei, dass der Rezipient solcher besonderen, gesammelten Gegenstände über das, was er sieht, hinausgeht und es mit vorgängigen Narrationen verknüpft. „[...], sie sind Vermittler zwischen dem Betrachter, der sie sieht, und dem Unsichtbarem, aus dem sie kommen" (Pomian 1998: 40).

12 Wir haben es also mit einer vermeintlichen Einheit Autor = Erzähler zu tun, wobei es sich nicht um ein autofiktionales Werk handelt, sondern die Autor-Erzähler-Einheit auf der Textebene inszeniert wird.

13 Die Theatralität des Bildbegriffs hat eine lange Traditionslinie, lässt sich jedoch maßgeblich auf Freuds Urszene beziehen, anhand derer sich über das Phantasma ein erweiterter und dynamischer Bildbegriff ableiten lässt, wie dies Andreas Wolfsteiner und Markus Rautzenberg tun (s. Anm. 26). 
diese Räume allerdings konkret funktionieren, wie sie zu solchen Widerlagern reflexiver oder auch subversiver Prozesse werden, bleibt bei Foucault im Unklaren. Mujica Láinez konstituiert das Museum ebenso als Raum der Reibung, in dem fixierte Vorstellungen von Kultur und Geschichte unterlaufen werden. Allerdings gesteht er dabei das subversive und innovative Potential nicht dem Raum als solchem zu; es bedarf eines Beobachtungs- und Handlungssubjekts sowie der narrativen Transformation und Weitergabe des Erlebten. Im Rezeptionsakt prallen kollektives Imaginäres als konservative Kraft, ${ }^{14}$ wie es die ausgestellten Werke repräsentieren, und die momenthafte Präsenz, die die Werke durch die Zugabe individueller Imagination gewinnen, aufeinander. Von der Subjektivität des Betrachters ausgehend aktualisieren sich die ausgestellten Ordnungen in der direkten Interaktion. Es entsteht ein unkalkulierbarer Überschuss an subjektivierter Bedeutungszuschreibung, der zum Geschoss, oder ,Widerlager ${ }^{6}$ werden kann.

Foucault selbst geht auf derlei Akte der Subjektivierung nicht ein. ${ }^{15}$ Dieser subjektive Erlebnisraum, in dem es zu Verschiebungen von der repräsentativen Kraft der Werke hin zu deren momenthafter Präsenz kommt, entsteht im Romangeschehen zudem jenseits der normalen Besuchssituation: Nachts, wenn die Türen für die Öffentlichkeit geschlossen sind, offenbaren die Figuren der Werke ihre geheime, zweite Existenz („vida oculta“, „existencia doble“, Mujica Láinez 1984: 10) nur einer einzigen und damit privilegierten Person (,un privilegio singular“ Mujica Láinez 1984: 10) - dem ,novelista‘, einem Fremden aus der Ferne („extranjero, habitante amistoso de un remoto país" Mujica Láinez 1984: 10). Für die PradoGesellschaft, die sich aus den Figuren und Szenerien der Gemälde und der Skulpturen zusammensetzt, wird die Nacht zum Tag. „[L]a otra vida de la pinacoteca“ (Mujica Láinez 1984: 66) beginnt. Tagsüber dazu verurteilt, still an ihrem vom Künstler gegebenen Platz zu verweilen, entwickeln sie des Nachts ein Eigenleben.

A poco que cae la tarde y que empieza a anochecer, los personajes de las pinturas y las estatuas del Museo del Prado, se desperezan y sacuden. Durante el día entero, permanecieron inmóviles, dentro de sus marcos o encima de sus pedestales, para admiración y tranquilidad de los turistas. Nadie, ni el estudioso más avizor, pudo advertir alguna mudanza en sus actividades a menudo embarazosas, tan habituados están a cumplir con la plástica tarea que les asignó la imaginación de sus creadores. ${ }^{16}$ (Mujica Láinez 1984: 9) ${ }^{17}$

14 Ich beziehe mich hier auf die Qualität des kollektiven Imaginären nach Castoriadis. Siehe Fußnote 6.

15 Die Qualität des subjektiven Rezeptionsvollzugs geht also über die Konzeption der Heterotopie im Sinne Foucaults, die sich grundlegend auf das Prinzip der Ordnung stützt, hinaus und weist in Richtung postcolonial studies, innerhalb derer die Performativität des Moments zentral ist. Fixierte Ordnungen werden als eine verwirklichte Möglichkeit von vielen herausgestellt und so eine Veränderung für Gegenwart und Zukunft als potentiell realisierbar dargestellt. „Wenn der Tag zu Ende geht und es anfängt, Nacht zu werden, strecken und recken sich die Charaktere der Bilder und Statuen des Prado und schütteln sich aus. Während des gesamten Tages verharren sie unbewegt in ihren Rahmen oder auf ihren Podesten, um der Bewunderung und Zufriedenstellung der Touristen willen. Niemand, auch nicht der achtsamste Beobachter, konnte einer Veränderung ihrer oftmals unangenehmen Beschäftigun- 
Die Bilder bzw. Bildwerke werden hier als eigentlich lebendig vorgestellt. Tagsüber stehen sie jedoch im Dienste ihrer Schöpfer, die sie mit einer speziellen Bedeutung und somit gesellschaftlichen Funktion belegt und fixiert haben. Die Rahmen und Podeste symbolisieren die Bannung der Bilder. Die suggerierte Normalität der ausgestellten Werke (,la normalidad de las obras expuestas“ Mujica Láinez 1984: 10) stellt eine Beruhigung (,tranquilidad') für die Besucher dar. Im institutionellen Raum des Museums bürgt die gesellschaftlich konventionalisierte Bedeutungszuschreibung als Norm für klare Strukturen und Vorstellungen geschichtlich fixierter Wirklichkeitskonstruktionen. In der Institution Museum herrschen Reglementierung und Regeltreue. Prinzipien von Ernsthaftigkeit und Mäßigung werden streng überwacht. Die Aufsichten gehen „rhythmischen Schrittes“ (,paso cadencioso") durch die Galerie, „[...] kontrollieren die Uhren; sie versichern sich des normalen Zustandes der ausgestellten Werke, sie überprüfen eine Ecke nach der anderen; sie unterhalten sich mit gedämpfter Stimme“ (,[...] controlan los relojes; verifican la normalidad de las obras expuestas, revisan rincón trás rincón; charlan en voz mesurada." Mujica Láinez 1984: 10).

Gegen die Statik der Museumswelt bei Tag setzt der Text kontrastiv den lauten Tumult, die Maßlosigkeit und überschäumende Lebendigkeit der Figuren bei Nacht, was auf die Unbändigkeit ikonischer Energie hinweist. Der sonst so ruhige Raum wird nun von lauten Geräuschen und tosendem Lärm erfüllt, wie etwa als sich aus dem Gemälde La disputa con los doctores en el Templo ${ }^{18}$ von Veronese lautstark und unter wütendem Geschrei ein Streit entfacht. „Ahora se han cerrado las puertas del Museo, y doquier sus secretos habitantes reviven. De la tela de Paolo Veronese se levanta una colérica gritería, como si audiblemente se prolongase la disputa que pintó el véneto" (Mujica Láinez 1984: 61). ${ }^{19}$

Der Museumsraum bei Mujica Láinez bestimmt sich aus der Reibung von Gegensätzen. Es kommt zu Brüchen und Zusammenstößen, die eine Spannung erzeugen und zu Irritationen führen. Dieses Phänomen zieht sich durch den gesamten Roman und prägt ihn maßgeblich. Eingefügt in diesen Spannungsraum ist eine Mittlerfigur: der ,novelista', der als ,transmisor (Übermittler) und ,testigo' (Zeuge) des geheimen, nächtlichen Lebens fungiert. Die Spaltung des ,novelista ${ }^{`}$ in ,Übermittler ${ }^{\varsigma}$ und ,Zeuge impliziert die Problematik von Fiktionalität und Faktualität. Der Zeuge und das Phänomen Zeugenschaft insgesamt werden - insbesondere in den Geschichtswissenschaften - mit Faktualität qua Wahrhaftigkeit assoziiert. Mu-

gen gewahr werden, so gewohnt sind sie es, ihre bildgegebene Aufgabe zu erfüllen, die ihnen die Imagination ihrer Schöpfer zugewiesen hat."

17 Das Werk liegt nur im spanischen Original vor; die Übersetzungen ins Deutsche stammen von der Verfasserin des Artikels.

18 Paolo Verones. La disputa con los doctores en el Templo. (Jesus unter den Doktoren im Tempel). Um 1560. Öl auf Leinwand. $236 \mathrm{~cm}$ x $430 \mathrm{~cm}$. Mueso Nacional del Prado.

19 „Nun sind die Türen des Museums geschlossen und überall erwachen seine heimlichen Bewohner zum Leben. Aus der Leinwand Paolo Veroneses erhebt sich ein wütendes Geschrei, als ob sich der Streit, den der Venezianer malte, hörbar verlängerte.“ 
jica Láinez' Text lebt aus einer Zeugenschaft der Anschauung, die sich hier durch eine starke, unmittelbare Erlebnisdimension auszeichnet. Doch ist der ,novelista' nicht nur ,Zeuge', der sich im Bereich des Faktualen bewegt, sondern auch ,Übermittler ${ }^{6}$ und zwar ein narrativer Übermittler, wodurch er in den Bereich der Fiktionalität eintaucht. Spannend ist im Bezug auf das Verhältnis von Faktualität und Fiktionalität hier insbesondere die Überlagerung beider Positionen. Diese zweifache Rolle des ,novelista' korreliert mit der doppelten Beschaffenheit des Museums: zum einen als Ort materieller Zeugenschaft, der dem Anspruch von Wahrhaftigkeit unterliegt, zum anderen als Ort kreativer Neuordnung, ein Möglichkeitsraum, der potentielle Narrationen enthält, die sich im Erleben dieses Raums durch ein Subjekt realisieren. Der ,novelista' bewegt sich also innerhalb eines solchen Spannungsraumes und trägt selbst eine ebensolche Spannung in sich.

,Transmisor ${ }^{6}$ und ,testigo' sind darüber hinaus beide informationstheoretische Begriffe. Sie verweisen auf die bedeutsame Position des, novelista' als Bote im Sinne Sybille Krämers, der auf die Funktion des Vermittelns und Übertragens in Bezug auf das Medium abhebt und damit dezidiert dessen Materialität übersteigt (vgl. Krämer 2008). Fragen nach der Art und Weise der Vermittlung sowie der Beschaffenheit des Kommunikationsaktes im Museum rücken in den Fokus. Wie erhält der ,novelista' Zugang zu der nächtlichen Welt? Wie sind seine Position und Relation zum Geschehen, zum Leben der Figuren bestimmt? Dies soll im nächsten Punkt beleuchtet werden, bei dem es um den Prozess der Übertragung und Umbildung von Bildern in deren Rezeption geht.

\section{Bilder treffen. Blicken und angeblickt werden}

Mit seiner textuellen performativen Bildbetrachtung bietet sich der Roman als ein Prozess der Bildaneignung dar. Dem ,novelista' kommt dabei die exponierte Rolle zu, zugleich Empfänger und Produzent von Bildern zu sein, was auf zwei Ebenen angesiedelt ist: Zum einen ist er passiver Zeuge (,testigo') der sich vor ihm entfaltenden Bildwelten; zum anderen gibt er diese Bilderfahrungen als aktiver Übermittler (,transmisor') narrativ weiter. Insofern die Bildbetrachtung immer schon die doppelte Disposition von aktiv und passiv beinhaltet, verweist sie auf ein wesentliches Merkmal des Performativen. ${ }^{20}$

Das Zusammenkommen von aktiv und passiv findet seinen Widerhall in der Idee von Blick und Gegenblick als einem Erklärungsmodell innerhalb der Bildwis-

20 Krämer stellt in Bezug auf die theatrale und kunsttheoretische Perspektive der performances deren doppelten Charakter heraus, der stets ,Tat' und ,Widerfahrnis', ,Machen' und ,Empfangen', ,Vollzug' und ,Entzug' bedeutet (Krämer 2009: 4). Sie bezieht sich hierbei maßgeblich auf Erika Fischer-Lichte, die auf dem Gebiet der Performativität in den Theaterwissenschaften und der Übertragung und Ausweitung zum kulturellen Phänomen und Theorem insgesamt, die zentrale Bezugsgröße ist (Fischer-Lichte 2001, Fischer-Lichte 2004). 
senschaften und bezieht sich auf die Eigenlogik und Wirkmacht von Bildern. Blicken meint hierbei in erster Instanz die Wahrnehmung eines Angeblicktwerdens, die sich beim Betrachter als Widerfahrnis äußert, auch wenn diese im eigenen Anblicken selbst entfacht wird; durch den eigenen Blick wirkt das Bild auf den Betrachter ein, tritt mit ihm in Kontakt. Dieses Angeblicktwerden aktiviert im Blickenden Bilder, Bildaktivitäten, die jenseits der visuellen Offensichtlichkeit der Bildoberfläche und des kontrollierten Wissens des Blickenden liegen. ${ }^{21}$ Die Wirkkraft jenseits der Logik des Intellekts bezeichnet Wulf als genuines Bildwissen, das in der ästhetischen Qualität der Erfahrung liegt. „Die Bilder wissen etwas von uns, was wir nicht wissen. Sie erhalten [sic] ein Element der Überraschung, das sich nicht voraussehen lässt und sich häufig der Alltagsrationalität entzieht, bevor sich uns der Sinn der Bilder erschließt" (Wulf 2005: 37). Im Blick liegt das Aktivierungspotential, das die Bilder animiert, ${ }^{22}$ sodass sie etwas in uns als dem Betrachter berühren: Eine Interaktion zwischen Bild-Körper ${ }^{23}$ und Betrachter-Körper setzt sich in Gang. Zentral ist, dass der Gegenblick des Bildes eine - im übertragenen Sinne - taktile Qualität besitzt. Der Gegenblick trifft einen als innere Berührung und löst ein Betroffen-Sein aus. „Indem ich den anderen sehe, objektiviere ich ihn; indem ich ihn anblicke oder von ihm angeblickt werde, fühle ich ihn; [...]" (Krämer 2009: 6). Sybille Krämer unterscheidet hier Blicken und Sehen dadurch, dass die Berührung dem Blicken als Modus des visuellen Kontakts vorbehalten ist. Krämer leitet diese Unterscheidung von Sartre her, auch wenn die Begriffe bei ihm im Kontext des zwischenmenschlichen Kontakts diskutiert werden und folglich eine andere Bedeutungsdimension entfalten. Krämer vollzieht nun aber den Brückenschlag zu den Bildwissenschaften und macht sich Sartres Unterscheidung zu Nutze, um der spezifischen Eigenlogik von Bildern auf die Spur zu kommen, die sie in deren Performativität sieht. In der Reziprozität des Blicks erkennt Krämer den „Nährboden für ein Verständnis der Performanz von Bildern“ (Krämer 2009: 7). Bei Mujica Láinez findet sich erstaunlicherweise sowohl die performative Kraft des Bildes, wie es ein Blick-Gegenblick-Modell nahelegt, als auch die qualitative Differenzierung visueller Modi narrativ ausgestaltet.

21 „Dieses Angeblicktwerden wird als ein Geschehen erfahren, bei dem das Bild ,zum Akteur wird, während dem Betrachter etwas widerfährt, das seiner Kontrolle nicht einfach unterliegt, obwohl er durch den Blick auf das Bild dieses Geschehen überhaupt erst evoziert" (Krämer 2009: 7).

22 „Die Medien selbst sind ein Archiv von toten Bildern, die wir erst in unserem Blick animieren" (Belting 2001: 214).

23 Die Körper-Metapher geht auf Belting zurück. Der Bild-Körper meint hierbei das Medium, in dem Bilder in Erscheinung treten. Der Körperbezug ist dabei ein doppelter: „Die Körperanalogie kommt in einem ersten Sinne dadurch zustande, daß wir die Trägermedien als symbolische oder virtuelle Körper der Bilder auffassen. Sie entstehen in einer zweiten Hinsicht dadurch, daß sich die Medien unserer körperlichen Wahrnehmung einschreiben und sie verändern. Sie steuern unsere Körpererfahrungen durch den Akt der Betrachtung in dem Maße, wie wir an ihrem Modell die Eigenwahrnehmung ebenso wie die Entäußerung unseres Körpers üben“ (Belting 2001: 13-14). 
Los turistas suelen estar distraídos; el tremendo cansancio los vence, [...]. Dóciles, mudos, fotografiantes, dejan vagar en torno los ojos fatigados. [...] Miran, miran, pero en la mayoría de las etapas, casi no ven. [...] Pero si las fuerzas le diesen para aguzar el interés y los ojos, tendría que percatarse el turista de que, sobre determinadas obras, se ha superpuesto una sutil, imprecisable veladura, que perturba vagamente las imágenes. ${ }^{24}(\mathrm{Mu}-$ jica Láinez 1984: 108-109)

Die Tagestouristen blicken nur (,miran') und sehen nicht (,casi no ven'). Im Spanischen werden die Begriffe also nun genau diametral verwendet: ,mirar, was mit ,blicken' zu übersetzen ist, steht für, sehen', und ,ver', was mit ,sehen' zu übersetzen ist, steht für ,blicken', in seiner kommunikativen Dimension. Die gegensätzliche Verwendung der Worte ist dabei nicht entscheidend, denn es geht um die generelle Differenzierung unterschiedlicher Qualitäten des visuellen Kontaktes, den Mujica Láinez in seinem Text entsprechend philosophischen und bildtheoretischen Konzepten vornimmt. Den Touristen bleibt die lebendige Welt der Bilder verschlossen, obwohl ihnen durchaus eine solche Möglichkeit der Erfahrung eingeräumt wird, würden sie ihre Augen ein wenig anstrengen („Pero si las fuerzas le diesen para aguzar el interés y los ojos"). Doch da sie die Bilder objektivieren, finden diese keinen Widerhall in ihnen - eine Kommunikation kann nicht zustande kommen.

Der ,novelista' hingegen erfährt die Bilder - in dieser sehr spezifischen Ausprägung von Erfahrungshaftigkeit liegt die ebenso signifikante Konstitution der Narration zu Grunde. ${ }^{25}$ Das Erfahren der Bilder in der Betrachtung ist zum einen als Widerfahrnis gekennzeichnet: Es stößt dem ,novelista' zu, denn er nimmt sich weder als aktiv Partizipierender noch als Urheber des Geschehens wahr. Die Vermutung, dass wir uns dennoch in seiner Erlebniswelt befinden, legt der Eröffnungsprolog nahe, in dem die Erzählsituation wie folgt beschrieben wird: „Y como es su oficio, el novelista cuenta aquí lo que vio y oyó “ (Mujica Láinez 1984: 11). („Und wie es seiner Aufgabe entspricht, erzählt der Erzähler hier, was er sah und hörte.") Der Eindruck einer Koppelung von passiver Widerfahrnis einerseits und aktivem Erlebnis andererseits wird durch die Erzählperspektive be-

24 „Die Touristen sind für gewöhnlich zerstreut; die enorme Müdigkeit besiegt sie, [...]. Fügsam, stumm, fotografierlustig lassen sie ihre müden Augen herumstreifen. [...] Sie schauen, sie schauen, aber die meiste Zeit, sehen sie fast nichts. [...] Aber wenn sie ihre Kräfte mobilisieren würden, um ihr Interesse und ihre Augen zu schärfen, müssten sich die Touristen bewusst werden, dass sich über bestimmte Werke ein feiner, nicht bestimmbarer Schleier gelegt hat, der die Bilder leicht stört."

25 Monika Fludernik entwickelt in Towards a,Natural ${ }^{\star}$ Narratology Erfahrungshaftigkeit (,experientiality') als tiefenstrukturelles Konzept des (fiktionalen) Erzählens. Als natürlicher Parameter menschlichen Seins, Bewusstseins und Handelns macht die Erfahrungshaftigkeit die mimetische Evokation der fiktionalen Welt möglich; klassische Charakteristika von Narrativität wie Handlung als kausale Ereignisfolge oder Zeitlichkeit leiten sich aus der Erfahrungshaftigkeit $a b$ und sind dieser nachgeordnet. Demnach ließe sich aus der Art von Erfahrungshaftigkeit auf Narrativität schließen und vice versa. Geeignet erscheint dieser postklassische Ansatz auch dadurch, dass sich Narrativität hier gerade nicht auf (fiktionale) Texte beschränkt, sondern auch auf die Bilderfahrung und narrative Umwobenheit von Bildern beziehen ließe (vgl. Fludernik 1996: insbes. 20-30). 
stätigt. Es handelt sich gerade nicht um einen autodiegetischen Erzähler, der eindeutig als Quelle der Erlebniswelt auszumachen ist. Stattdessen haben wir es mit einem heterodiegetischen Erzähler zu tun, der damit nicht direkt in die Handlung involviert ist, aber dennoch in Verbindung mit einer internen Fokalisierung seine Innenwelt als den Ursprung des Erzählten darstellt. Die Bilder werden zu einem theatralen Szenario, das der Betrachter als von ihm abgekoppelt wahrnimmt, obwohl es eigentlich ihm selbst bzw. seiner Phantasie entspringt. ${ }^{26}$ Die Phantasie (oder Einbildungskraft, Imagination ${ }^{27}$ ) spielt bei diesem Prozess einer Verinnerlichung der Bilder eine zentrale Rolle.

Phantasie macht es möglich, Bilder wahrzunehmen, auch wenn das Abgebildete nicht anwesend ist. Sie ermöglicht das innere Sehen, das Erinnern und das Entwerfen von zukünftigen Handlungen. ${ }^{28}$ [...] Die Phantasie hat eine chiastische Struktur, in der sich innen und außen kreuzen. (Wulf 2005: 43)

Im Text besetzt die Phantasie die Schlüsselstelle, um äußere in innere, mentale Bilder zu überführen. Die entstehenden nächtlichen Bildwelten sind ein feines Gespinst, gesponnen aus den immateriellen und ephemeren Fäden der Phantasie:

El novelista se retrasó, porque esperaba detenerse frente a los Velázquez, en un ámbito semivacío; y, en efecto, se desvanecieron los caminadores y se acallaron los murmullos. Entonces quien esto escribe, avezado por su privilegiada y mágica situación, capta algo como un palpitar que conmueve la serenidad majestuosa de las obras de arte. Y comprueba que de algunas de ellas se desprende una levísima tela de araña, que al flotar en el aire asume la forma ingrávita y diáfana de un corcel, de unas liebres, de un perro, de un ondulante dragón. Todo ese inmaterial entrelazarse de diseños, gira, vacila y termina por posarse donde exactamente le corresponde. ${ }^{29}$ (Mujica Láinez 1984: 110)

26 Einen solchen theatralen Bildbegriff, der sich als Szenario vor den Augen eines Subjekts vollzieht und in das es als eigentlicher Urheber involviert ist, ohne sich jedoch eines direkten Einflusses auf das Geschehen bewusst zu sein, finden Markus Rautzenberg und Andreas Wolfsteiner in Freuds Begriff des ,Phantasmas'. „Das Phantasma als Bild ist weder innen noch außen, weder image noch picture und durch eine spezifische Form der Subjektpartizipation charakterisiert, [...]" (Wolfsteiner/Rautzenberg 2014: 24).

27 Die drei Begriffe Einbildungskraft, Imagination (lat. imaginatio) und Phantasie (gr. phantasia) konvergieren in wesentlichen Aspekten, da sie im Kern auf das Gleiche referieren. Sie sind allerdings kulturell und historisch unterschiedlich verortet und haben dadurch jeweils verschiedene Akzentuierungen und Bedeutungsnuancierungen erfahren. (Metzler Lexikon Ästhetik 2006: 89)

28 Indem Phantasie sowohl zeitlich zurück, als auch nach vorn ausgerichtet ist und damit Vergangenheit, Gegenwart und Zukunft zusammenschließt, birgt sie das Potential, in der Evokation und Reflexion von Vergangenem, aus dem Moment der Gegenwart heraus, Zukunft zu gestalten. In Zusammenhang mit der postkolonialen Konstellation, Lateinamerika-Europa, in der der Text steht, gewinnt dieser Aspekt an Wichtigkeit, da durch Phantasie geschichtliche Konstruktionen als perspektivisch veränderbar erscheinen. „Der Schriftsteller blieb zurück, denn er beabsichtigte, in einem halbleeren Raum vor den [Bildern] Velázquez' zu verweilen; in der Tat verschwanden die Herumlaufenden und das Gemurmel verstummte. Nun denn, wer dies hier schreibt, was er durch seine privilegierte und magische Situation erfährt, nimmt etwas wie ein Pochen wahr, das die majestätische Stille der Kunstwerke bewegt. Und er stellt fest, dass sich von einigen von ihnen ein ganz 
Das ,Lebendig-werden' der Bilder beginnt mit einem Pochen (,un palpitar'), das aus dem Feld der Körpermetaphern entlehnt ist und das Pochen des Herzens evoziert. Die Bilder, im Blick des Betrachters aktiviert, erwachen in ihm zum Leben. Beide - Bild und Betrachter - sind durch ein körperhaftes Beben verbunden und in der Phantasie entspinnen sich flüchtige Zeichen (,diseños'), die sich miteinander verflechten (,entrelazarse). Dieses Verweben von Zeichen zeigt in Richtung Sprache und damit hin zum Text, zur Narration als einer Konfiguration von Bildzeichen. Innerhalb eines solchen Prozesses des Aufbrechens und Neukonfigurierens der Bildelemente bekommen diese eine aktuale narrative Ordnung eingeschrieben. In dieser narrativen Verknüpfung erlangen die Ausstellungsstücke eine momenthafte Temporalität. Der Museumsraum wird im Rezeptionserleben um die Dimension der Zeit erweitert.

Die Betrachtung der Bilder ist Teil eines inneren Prozesses, des Blickaktes selbst, und wird dennoch als ein äußeres Erleben wahrgenommen. „El novelista no se le despega a Velázquez [...]. Lleva la audacia hasta la familiaridad de aspirar a ponerle una mano encima del hombro, y al intentarlo comprueba que sus dedos vacilan, cerrados sobre la transparencia del aire" (Mujica Láinez 1984: 90). ${ }^{30}$ Der ,novelista' nimmt sich mitten im Geschehen wahr, er ist ergriffen, berührt von der Macht der Bilder, die sich performativ in der Responsivität des Blicks entfaltet. ${ }^{31}$ Doch bei der tatsächlichen Berührung mit dem sich vermeintlich bei ihm befindlichen Velázquez ist dort nichts als Luft. Das Empfinden eines inneren Berührtwerdens korrespondiert gerade nicht mit einer tatsächlich taktilen Berührung. Wulf spricht in Bezug auf die Bildrezeption von ,mimetischen Prozessen', die sich durch eine starke Sinnlichkeit auszeichnen.

Mimetische Prozesse sind performativ; sie sind sinnlich auf Bilder als Bilder fokussierte Prozesse der Weltaneignung und Welterweiterung. In mimetischen Prozessen stellt die Phantasie die Verbindung zur Welt her und macht Außenwelt zur Innenwelt und Innenwelt zur Außenwelt. In diesen Prozessen spielt das Begehren, sich die Welt anzueignen, eine konstitutive Rolle. In diesem Begehren erzeugen nicht nur wir die Bilder, sondern auch die Bilder uns. Die Begehrensstruktur unseres Körpers und unserer Wahrnehmung bewirken, dass, bevor sich unsere bewusste Wahrnehmung auf die Dinge richtet, die Dinge uns ansehen. (Wulf 2005: 39)

leichtes Spinnennetz ablöst, das in der Luft schwebend die schwerelose und durchscheinende Form eines Roßes, einiger Hasen, eines Hundes, eines wellenförmigen Drachens annimmt. All dieses immaterielle Verflechten von Umrissen dreht sich, schlingert und setzt sich schließlich genau dort nieder, wo es ihm entspricht."

30 „Der Schriftsteller trennt sich nicht von Velázquez [...]. Er ist so kühn, in einer vertraulichen Geste danach zu trachten, ihm eine Hand auf die Schulter zu legen und als er es versucht, stellt er fest, dass seine Finger sich zögernd über der durchsichtigen Luft schließen.“

31 „Zugleich eröffnet der Doppelcharakter des Sehens in der Responsivität des Blicks, mit dem das Bild sich an den Betrachter wendet, eine Dimension der Taktilität und des Fühlens, die den Weg aus dem Paradoxon weist, dass das Bild in seiner Sichtbarkeit von etwas Unsichtbarem und auch Unergründlichen zehrt, das uns - wiewohl nicht gesehen gleichwohl ergreifen kann“ (Krämer 2009: 14). 
Die hier angesprochene „Begehrensstruktur unseres Körpers“ verweist auf besagte Performativität der Bilder, die einen doppelten Selbstbezug - nach außen und nach innen - meint. Im performativen Akt der Bildbetrachtung kommt es zum Zusammenfließen von kollektivem Imaginären und Imagination. Die Performativität des Bildlichen könnte als revelatorische Instanz sowohl hinsichtlich gesellschaftslogischer ${ }^{32}$ wie identitätslogischer Strukturierungen gewertet werden. Die Qualität der Bilder, die sich in der Interaktion zwischen dem ,novelista' und den Bildwerken im paradigmatischen Raum des Prados abzeichnet, ließe damit Rückschlüsse auf dessen Subjektpositionierung innerhalb der spezifischen gesellschaftspolitischen Situation einer postkolonialen Konstellation zu, innerhalb derer er verortet ist. Dies soll im nächsten Punkt anhand der Narrativierung der eigenständigen Wirkkraft und Performativität der Bilder nachvollzogen werden.

\section{Die Präsenzerfahrung der Bilder. Ein Spektakel der Körper und Töne}

Wahrnehmen ist nach Ludger Schwarte eine körperliche Handlung, die sich grundlegend an „raumbildende Prozesse und Interaktionen mit den Dingen“ knüpft (Schwarte 2005: 279). Wie zuvor ausgeführt, ist der Körper des ,novelista Quelle und Austragungsort der theatralen Aktualisierung der Bilder bei deren Betrachtung. Doch ist nicht nur für ihn die Bildbetrachtung eine körperhafte Erfahrung. Bei ihrer Transformation in den Text entwickeln die Bilder eine ganz eigene und eindringliche körperlich-sinnliche Qualität, die zum einen thematisch, zum anderen formal evoziert wird.

Diese Körperhaftigkeit entfaltet sich aus der präsentischen Aktualisierung der Bildwerke im Blick des ,novelista' und kontrastiert stark mit deren repräsentativen Eigenschaften. Damit folgt die Theatralisierung der Bilder, wie sie sich im Text darstellt, ebenfalls dem Prinzip eines kontrapunktischen Aneinanderstoßens von Gegensätzen entlang der Leitlinie von Repräsentation und Präsenz. Im Zeichen der Repräsentation stehen detailreiche Schilderungen aufwendiger Kleider und Kostüme, Rüstungen und Ausrüstungen, Szenerien und Kulissen, wie sie sich aus der Sichtbarkeit der Gemälde ableiten. Sie verweisen symbolisch auf die historischsozialen Rollen ihrer Träger. Eine Parade an adligen Herrschaften bietet sich dar:

Carlos V, [...], con un poco más de treinta años y el traje lució de Bolonia para ceñir la corona lombarda; lo acompaña Sampere, su querido sabueso irlandés; luego Felipe II,

32 Christoph Wulf und Jörg Zirfas verweisen auf die doppelte Gerichtetheit der Performativität kulturellen Handels, einerseits Bilder zu erzeugen und gleichzeitig von Bildern erzeugt zu sein. Im Anschluss ließe sich umgekehrt folgern, dass sich aus der Performativität des Bildes auf kulturelle performative Strukturen rückschließen ließe (vgl. Wulf/Zirfas 2005: 7).

Krämer hebt ebenfalls darauf $\mathrm{ab}$, dass sich das performative Betrachten von Bildern als „kulturimprägnierte und kulturstiftende Handlung“ werten lässt (vgl. Krämer 2009: 16). 
joven príncipe todavía, negra y áurea la cortesana armadura, admirable el diseño de las piernas, [...]. [...] la Emperatriz cuya falda hace pompa, [...]. ${ }^{33}$ (Mujica Láinez 1984: 42)

In frappierendem Kontrast zu der äußeren Beschreibung stehen deren Auftreten, Handlungsweise und Charakter. Fallen die Figuren des Nachts aus ihren Rahmen, fallen sie aus ihren Rollen. ${ }^{34}$ Die ehrwürdigen Posen werden zur Posse. Sie verlassen den im künstlerischen Medium fixierten, zugewiesenen Platz in der Geschichte. Denn versteht man nach Belting ${ }^{35}$ Trägermedien als Ausdruck symbolischer Handlungen, dienen die im Prado ausgestellten Gemälde und Skulpturen der Manifestation bestimmter Wissens- und Machtordnungen. Mit dem Verlassen der Rahmen sind die Figuren ihren repräsentativen und restringierenden Aufgaben entbunden und stehen nun im Zeichen der Präsenz ihrer aktuellen Realisierung im Akt der Betrachtung. Die Evokation von Gegenwärtigkeit, die zu Reibungen und Brüchen führt, wird insbesondere über Töne und Geräusche sowie eine starke Körperlichkeit geleitet. ${ }^{36}$ Eine tosende Geräuschkulisse erhebt sich, aus Lärm und Geschrei und Lachkaskaden: „Paco ríe, y sus carcajadas hacen vibrar los vidrios del techo“ (Mujica Láinez 1984: 18). ${ }^{37}$ Tonalitäten nuanciertester Qualität „Cantan, gor-

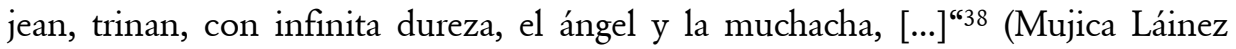
1984: 20), aus Lauten des menschlichen Körpers, wenn etwa die Figuren aus Velázquez’ Gemälde „schreien“, („gritan“) und „stammeln“ („tartajea[n]"); nicht selten produzieren die Figuren Laute, die tierische Qualitäten annehmen, wenn sie „gackern“ („cacarea[n]“) und brüllen wie Löwen („ruge[n]“) (Mujica Láinez 1984: 52).

Allein durch diese exaltierte Lautlichkeit gewinnen die Figuren schier spürbar an körperlicher Materialität. Weiter steigert sich dieser Eindruck in Beschreibungen von Nacktheit und exzentrischen Posen, etwa, wenn Bacchus „sus carnes flo-

33 „Karl V., nicht der des Reiterstandbildes von Mühlberg, der fast Fünfzigjährige, sondern der, der sich stehend verewigt, mit etwas mehr als dreißig Jahren und der Tracht von Bologna, um sich die lombardische Krone aufzusetzen; er ist von Sampere begleitet, seinem geliebten irischen Schweißhund; dann ist da noch Philipp II., der noch junge Prinz, in höfischer Rüstung, schwarz und golden, bewundernswert ist die Modellierung seiner Beine [...]. [...] die Kaiserin, mit prunkvollem Rock, [...].“

34 Auf den Zusammenhang zwischen dem (Aus)Bruch der Bildfiguren, des Verlassen des Rahmens und dem Bruch mit sozialen wie intellektuellen Kategorien, wodurch die gesellschaftliche, ideologische Struktur destabilisiert wird, weist Rachel Schmidt in ihrem Aufsatz hin (Schmidt 2003).

35 Vgl. Belting 2001: 50.

36 Seit Descartes hat sich das Auge als eine Art abstrakter Tastsinn stark von der Welt der Sinne distanziert, wodurch das Sehen von anderen sinnlichen Erfahrungen stark unterschieden wird. Die Bedeutungssteigerung des Lautlichen und Körperlichen bei der Bildbetrachtung, wie sie Mujica Láinez narrativiert, unterminiert als Erfahrung nun diese Differenzierung einer dominanten westlichen Denktradition. In der postkolonialen Konstellation, in der der Roman steht, bedeutet dies einen weiteren Aspekt, durch den die Hegemonie europäischer Denk- und Wissenstraditionen ironisch unterlaufen und in Frage gestellt wird.

37 „Paco lacht und sein Gelächter lässt das gläserne Dach vibrieren.“

38 „Sie singen, trillern, trällern mit unendlicher Beharrlichkeit, der Engel und das Mädchen, $[\ldots] . “$ 
jas, enormes tan totalmente desnudas [...]“39 (Mujica Láinez 1984: 15) zurücklehnt oder aber wenn er die , lüsterne Nymphe‘ (,la ninfa salaz`) wegschiebt, „y ríe con cada arruga, con cada frunce, cada bolsa, cada revolucionaria tripa; ríe con la plentitud de su voluminosa y desnudísima desnudez" (Mujica Láinez 1984: 20). ${ }^{40}$ Der Körper erfährt hier eine Personalisierung und Wahrnehmungsintensivierung. Die Falten von Bacchus' Körper lachen aus sich heraus und werden in der dreimaligen Variation des Begriffs fast wie Individuen dargeboten, bis das Lachen schließlich in den ,revolutionären Gedärmen' ankommt und mit der ,nacktesten Nacktheit' seinen absurden Kulminationspunkt erreicht.

Repräsentation und Präsenz prallen aufeinander und provozieren eine Kollision. Die derben, burlesken, lauten und lasziven Züge, die die Bildfiguren annehmen, brechen mit der Erhabenheit, Feierlichkeit und Ernsthaftigkeit, die den Kunstwerken im kontextuellen Rahmen der Gemäldegalerie des Prados zugewiesen werden und die deren repräsentativen Status als bildliche Dispositive von Machtstrukturen verbürgen sollen.

Verschärft werden diese Friktionen weiter durch die behandelten Themen und die sprachlichen Mittel. Die eigentliche Beschreibung und Interpretation der Gemälde und Skulpturen nach kunstgeschichtlichen Gesichtspunkten bleibt aus. Die Bildfiguren werden eigenständig und in ein buntes Spektakel von Alltagszenen aufgelöst, bei dem sich alles um die Befindlichkeiten, Sorgen und Probleme, Animositäten, Eitelkeiten und Eigenheiten der Figuren dreht. Bei allen - egal ob historischer König, römische Gottheit oder christliche Engelsfigur - stehen Charakter und Gefühlswelt im Mittelpunkt. ${ }^{41}$ „Luis XIV el Rey Sol de Rigaud, que oficialmente revestido de una armadura luminosa, colosal el pelucón y alígera la decorativa faja, asoma su carota de viejo irascible, y clama contra la iniquidad e inmoralidad de la resolución, [...]“(Mujica Láinez 1984: 58).42 „Júpiter, que detesta la grosería, se ofende y reclama cordura, con lo que el vallisoletano, sobreponiéndose, se inflama"“3 (Mujica Láinez 1984: 52) und selbst die Engel fühlen sich beleidigt und empfindlich getroffen, als der Zwerg Don Diego ihnen in seiner Märchenaufführung die Rolle der Feen auferlegen möchte. „Tuvo Don Diego

39 „sein schlaffes Fleisch, sein gewaltiges, ganz und gar nacktes Fleisch [...].“

40 „und mit jeder Falte, mit jeder Faltung, jeder Stülpung, mit jedem revolutionärem Bauch, mit der Gesamtheit seiner voluminösen und nacktesten Nacktheit [lacht]."

41 Mujica Láinez schließt hier an eine lange Tradition der Verlebendigung und Psychologisierung als Bildbeschreibungsverfahren an, wie sie bereits in den ekphrastischen Texten der (Spät-)Aufklärung etabliert sind. Im deutschsprachigen Raum ist Wilhelm Heinse mit seinem Ardinghello (1787) literarischer Vorläufer und Ausnahme zugleich, denn sein vermittelter Blick auf die Kunst ist ein dezidiert erotisierender und versinnlichender.

42 „Ludwig der XIV., der Sonnenkönig, von Rigaud, offiziell bekleidet mit einer strahlenden Rüstung, gewaltig ist die Perücke und gelockert die dekorative Schärpe, zeigt seine Grimasse des jähzornigen Alten und ereifert sich gegen die Ungerechtigkeit und Unsittlichkeit der Resolution, [...].“

43 „Jupiter, der Unhöflichkeit verachtet, ist gekränkt und fordert Vernunft, woraufhin sich der aus Valladolid überwindet, in Wut gerät und kräht." 
que resignarse y reducirlas a dos, puesto que los ángeles, ofendidos en masa, rechazaron el fascinador papel, [...]" (Mujica Láinez 1984: 74). ${ }^{44}$ Schon bei diesen kurzen Schilderungen zeigt sich, dass dem Roman vor allem ein ironischer Ton eigen ist. ${ }^{45}$ Die grotesken Situationen werden zum Teil bis ins Absurde gesteigert, wodurch die Figuren überspannt bis exzentrisch wirken.

\section{Zusammenschau und Ausblick}

In der theatralen Aktualisierung der Bilder im Anblick des ,novelista' kommt es, wie gezeigt, zu zahlreichen Ebenenbrüchen. Ein intensives Erleben der Figuren durch tonale und körperliche Qualitäten führt, ebenso wie die Alltagsthematik und die ironisch-groteske Rhetorik, zu Kollisionslinien zwischen Repräsentation und Präsenz. Diese Kollisionen werden als Reibung zwischen den in den Bildern repräsentierten Rollen der Figuren entlang gesellschaftlicher Diskurse ${ }^{46}$ und deren Aktualisierung im Akt der Bildbetrachtung wahrgenommen. Zwar ist ihre Kennzeichnung über äußere Attribuierungen ersichtlich, doch bleibt ein narratives Ausfüllen dieser Positionen aus. In der Evokation eines inneren und körperlichen Eigenlebens der Figuren im Hier und Jetzt der entfachten Phantasie des Betrachters werden soziale Rollen, Machtgefüge und Kategoriensysteme unterlaufen und in ihrer künstlichen Konstruiertheit vorgeführt, was gerade hinsichtlich der postkolonialen Konstellation des Textes wichtig ist. Europäische Hegemonieansprüche werden unter dem ironischen Blick des, novelista' infrage gestellt. Der Museumsraum konstituiert sich als Bühne der Aushandlung gesellschaftlicher und geschichtlicher Gefüge über den Akt einer individuellen performativen Bildbetrachtung.

Im Text realisiert sich die performative Dimension der Bildrezeption über ein stark synästhetisches Schreiben, das insbesondere im auditiven Bereich wirkt. Sprache und Narration erfahren hierbei eine sinnliche Erweiterung. Im Text wird Sinn durch Sinnlichkeit evokativ hervorgerufen; der Text imitiert dadurch Charakteristika und Strategien der Sinnstiftung, wie sie eigentlich im Bereich des Bildlichen zu finden sind. Mujica Láinez' Roman wird damit auf eine allgemeine ,ästhetische Dimension des Performativen" hin transzendiert, die nicht allein in Sprache aufgehen kann, sondern der Zugabe von Bild, Ton, Geschmack oder Geruch bedarf (vgl. Wulf 2005: 35) und hier beim Rezipienten allein sprachlich evoziert wird. Der Text funktioniert folglich zum einen über eine intensive Sinnlichkeit und zum an-

44 „Don Diego musste sich damit abfinden und sie auf zwei reduzieren, da die in Scharen beleidigten Engel die faszinierende Rolle ablehnten, [...].“

45 Nicht umsonst zieht Rachel Schmidt eine Parallele zum Esperpento Valle-Incláns, bei dem die Figuren wie in einem Zerrspiegel erscheinen und Lächerlichkeit und Groteske preisgegeben werden (vgl. Schmidt 2003: 123).

46 Es geht hier nicht allein um die gesellschaftlichen Rollen der einzelnen Bildfiguren, die sie im Kontext der Bilddiegese übernehmen, sondern um gesellschaftliche Funktionalisierung und Instrumentalisierung der Bilder in ihrer Gesamtheit, als deren Teil die auf ihnen dargestellten Figuren beschrieben werden können. 
deren über starke Kontrastierungen, durch die es zu besagten ironischen Brüchen kommt. Diese Textstrukturen die die Rationalität des Intellekts zu überschreiten suchen, rufen im Akt des Lesens Irritationen - und demnach ebenfalls sinnliche Reaktionen - hervor, die ihre Wirkung entfalten und Reflexionen hinsichtlich der Sinn- und Wahrhaftigkeit äußerlich fixierter Vorstellungen in Zweifel ziehen.

Im Text kommt es demzufolge zu einer Dekonstruktion fixierter, repräsentativer Gesellschaftsvorstellungen, die an einem europäischen Modell orientiert sind, ohne dass ein konkretes Gegenmodell aufgebaut wird. Eine gewisse Sinnstiftung deutet sich in der Konfiguration der Bilder als Choreografie an, die als Netze flüchtiger Bildzeichen verweben und der im Blick des Betrachters aktivierten Phantasie entspringen. Eine bleibende und beständige Ordnung allerdings zeichnet sich nicht ab. Die belebten Figuren lösen bei Anbruch des Tages ihr theatrales Treiben auf und kehren an ihre alten Positionen zurück. Der Leser wird in der Schwebe gehalten und in einem Zustand der Irritation entlassen.

Es lassen sich nun Kernaspekte der gewonnen Erkenntnisse hinsichtlich der Konstitution, Bedeutung und Funktion von Bildern auf die Problematik von Faktualität und Fiktionalität anwenden. Denn der Versuch einer Kategorisierung von Bildern ist - wie in der Einleitung kurz angerissen - aufs Engste mit besagter Thematik verknüpft. Bilder sind mehr als die Sichtbarkeit ihrer visuellen Oberfläche. Sie haben eine dualistische Anlage aus Repräsentation, deren historisch kultureller Gebundenheit und aktueller Präsenz. Bilder sind in den pragmatischen Vollzugsakt ihrer Rezeption eingebunden, innerhalb dessen sich ihr Dualismus durch das Zusammenfließen von kollektiven Imaginären und (individueller) Imagination äußert. Die spezifische Eigenlogik der Bilder wurzelt in deren Performativität, wie sie von Krämer anvisiert wird (Krämer 2009). Performativität liegt ihrem Wesen nach jenseits hierarchischer Strukturen wie wahr und falsch, da sie ihre Bedeutung im Moment der Handlung selbst entfaltet. ${ }^{47}$ Als Emergenzphänomen wurzelt in ihr das Potential zu Veränderungen hinsichtlich der Wahrnehmung von Vergangenheit, Gegenwart und Zukunft. Im Text von Mujica Láinez entspringt eine solche Wirkmacht aus der performativen textuellen Bildbetrachtung: In dem reziprok angelegten Rezeptionsakt aus Blick und Gegenblick entwickeln die Bilder - einst durch kontextuelle Rahmungen gebannt - im Rezeptionssubjekt eine neue, aktuelle Realisierung. Im Text werden Elemente des unsichtbaren Umraums, der jedes Bild umgibt, sichtbar.

Bei der Transformation der Bildbetrachtung in den Text kommt es zu einem medialen Sprung vom bildlichen zum textuellen Erleben, bei dem sich die im Rezeptionsakt erlangte Aktualität der Bildwerke als eine synästhetisch-sinnliche Intensität in den Text übersetzt. Als körperliche Reaktion der Irritation entfaltet sie im Leser ihre Wirkung. Sinn ist hier an Sinnlichkeit, körperhaftes Erleben ge-

47 John Austin stellt heraus, dass performative Sprechakte sich nicht an den Kriterien wahr oder falsch bemessen lassen (vgl. Austin 1962). 
bunden und subvertiert damit westlich geprägte Ratio als privilegiertes, aber einseitiges Modell der Weltsicht. Repräsentation und Präsenz hybridisieren in einem Moment spannungsvoller Reibung. Faktualität und Fiktionalität ließen sich nun, den in Bezug auf das Bild bereits diskutierten Oppositionspaaren von Sichtbarkeit und Unsichtbarkeit, Repräsentation und Präsenz analog beiordnen. Diese kategorialen Unterscheidungen halten konkreten Subjekthandlungen - wie Akte der Bildrezeption - nicht stand und zerschellen an der Performativität des Bildes.

Entscheidend für solche Amalgamierungsprozesse ist die Imagination oder Phantasie als Modus der Welterfassung und Weltaneignung, in der binäre Gegensätze zusammenfließen und sich emergente Neuordnungen bilden. Letztlich lassen Bilder sich nicht trennscharf in die Kategorien faktual versus fiktional einordnen. Sie sind Hybridisierungs- und Anlagerungsphänomene, die über Oppositionen wie faktual und fiktional funktionalisiert und, um in der Sprache des Gedankengebäudes dieses Artikels zu bleiben, gerahmt werden, um Wissen, Macht und Identitäten zu strukturieren und zu verhandeln.

\section{Literatur}

Austin, John (1962) How to Do Things With Words. The William James lectures delivered at Harvard University in 1955. Oxford: The Clarendon Press.

Belting, Hans (1990) Bild und Kult. Eine Geschichte des Bildes vor dem Zeitalter der Kunst. München: Beck.

- (2001) Bild-Anthropologie: Entwürfe für eine Bildwissenschaft. München: Fink.

Benjamin, Walter (1996) Das Kunstwerk im Zeitalter seiner technischen Reproduzierbarkeit: drei Studien zur Kunstsoziologie [1963]. Frankfurt a. M.: Suhrkamp.

Boehm, Gottfried (1978) „Zu einer Hermeneutik des Bildes“. Seminar. Die Hermeneutik und die Wissenschaften. Hgg. Hans-Georg Gadamer und Gottfried Boehm. Frankfurt a. M.: Suhrkamp. 444-71.

- (2001) Hg. Was ist ein Bild? [1994]. München: Fink.

Castoriadis, Cornelius (1990) Gesellschaft als imaginäre Institution: Entwurf einer politischen Philosophie. Frankfurt a. M.: Suhrkamp.

Didi-Huberman, Georges (1999) Was wir sehen blickt uns an: Zur Metapsychologie des Bildes. München: Fink.

Fischer-Lichte, Erika, und Christoph Wulf (2001) Hgg. Theorien des Performativen. Paragrana. Internationale Zeitschrift für Historische Anthropologie. Band 10, Heft 1. Berlin: Akademie Verlag.

Fischer-Lichte, Erika (2004) Ästhetik des Performativen. Frankfurt a. M.: Suhrkamp. Fludernik, Monika (1996) Towards a ,Natural' Narratology. London: Routledge.

Foucault, Michel (1991) „Andere Räume“. Aisthesis: Wahrnehmung heute oder Perspektiven einer anderen Ästhetik. Hg. Karlheinz Barck. Leipzig: Reclam. 34-46. 
Hutcheon, Linda (1988) A Poetics of Postmodernism: History, Theory, Fiction. London/New York: Routledge.

Krämer, Sybille (2008) Medium, Bote, Übertragung: Kleine Metaphysik der Medialität. Frankfurt a. M.: Suhrkamp.

- (2009) Gibt es eine Performanz des Bildlichen? Reflexionen über Blickakte <http:// userpage.fu-berlin.de/ sybkram/media/downloads/Performanz_des_Bildlichen .pdf $>$ (Zuletzt abgerufen am 09.03.14).

Mujica Láinez, Manuel (1984) Un novelista en el museo del Prado. Barcelona: Editorial Seix Barral.

Pomian, Krzysztof (1998) Der Ursprung des Museums: Vom Sammeln [1988]. Berlin: Wagenbach.

Sachs-Hombach, Klaus (2009) Hg. Bildtheorien: Anthropologische und kulturelle Grundlagen des Visualistic Turn. Frankfurt a. M.: Suhrkamp

Schmidt, Rachel (2003) „Hacer que los cuadros hablen: la problemática del marco en ,Un novelista en el Museo del Prado', de Manuel Mujica Láinez“. Revista Canadiense de Estudios Hispánicos. Reproducciones y presentaciones. Diálogos entre la imagen y la palabra. 28.1: 119-35.

Schwarte, Ludger (2005) „Das Einräumen von Bildlichkeit: Wahrnehmungshandlungen und Ausstellungsarchitektur". Ikonologie des Performativen. Hgg. Christoph Wulf und Jörg Zirfas. München: Fink. 279-99.

Wulf, Christoph (2005) „Zur Performativität von Bild und Imagination: Performativität - Ikonologie/Ikonik - Mimesis“. Ikonologie des Performativen. Hgg. Christoph Wulf und Jörg Zirfas. München: Fink. 35-49.

Wulf, Christoph und Jörg Zirfas (2005) „Bild, Wahrnehmung und Phantasie: Performative Zusammenhänge“. Ikonologie des Performativen. München: Fink. 732.

Wolfsteiner, Andreas und Markus Rautzenberg (2014) „Trial-and-Error-Szenarien. Zum Umgang mit Zukünften“. Trial and Error: Szenarien medialen Handelns. Paderborn: Fink. 7-29.

Metzler Lexikon Ästhetik: Kunst, Medien, Design und Alltag (2006) Hg. Achim Trebeß. Stuttgart/Weimar: Metzler. 
